\title{
Light forms tiny 3D structures
}

Hiroaki Misawa and Saulius Juodkazis

Photo-polymerization and dielectric breakdown using laser pulses can fabricate 3D nano- and micro-structures.

The current trend toward miniaturization in science and technology is sustained mainly by planar two-dimensional processing of materials. A shift to inherently-3D fabrication could increase the efficiency and functionality of micro-chips. The most promising 3D processing tool that is capable of sub-micrometer resolution is a tightly-focused laser beam. Such a beam can deliver energy not just to a material's surface, but inside its volume. 3D photo-modification of materials depends on processes that uses nonlinear optical absorption phenomena, the strongest of which is two-photon absorption.

Here, we discuss two methods of 3D photo-structuring: photopolymerization, and dielectric breakdown using femtosecond laser pulses that have been focused so tightly that the spot size is comparable to the wavelength. 3D photo-polymerized resists and resins can achieve features sizes smaller than 100nm. ${ }^{1,2}$ The critical exposure necessary to polymerize the material can accumulate either by the serial method of direct laser writing or by the parallel method of holographic patterning. In the direct-laser-writing method (see Figure 1) ${ }^{3-5}$ multiple laser pulses provide the energy for nonlinear absorption. The holographic method depends on interference between several beams and pulses (see Figure 2). 6,7 Another parallel method uses a microlens array with direct laser writing to create multiple beam foci. ${ }^{8}$

Holographic recording allows control of the phases and polarizations of interfering beams and is a versatile method for forming complex 3D lattices. ${ }^{9,10}$ The fabrication possibilities added by controlling the polarizations of interfering beams allows this method to form optically-active photonic structures or their templates. Some possibilities, shown in Figure 3, include chiral patterns made using three interfering side-beams or patterns exhibiting a photonic bandgap (the diamond-like pattern formed by four beams). The interference between six side beams and a central circularly-polarized beam can form spiral patterns. By adding a counter-propagating circularly-polarized beam, the interference forms a 3D pattern of ring-like structures that may be useful for studying left-handed materials. Note that $1 \mathrm{D}$ photonic structures can also be formed by interfering one circularly-polarized side-
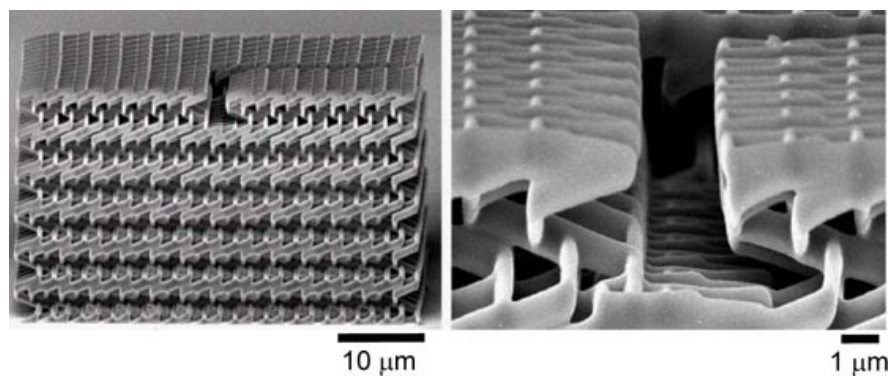

Figure 1. Two views of a spiral photonic-crystal template that includes an intentional defect in the top layer. The photonic crystal was recorded in SU-8 resist using direct laser writing at a wavelength of $800 \mathrm{~nm}$, with $0.5 \mathrm{~nJ}$ pulses $180 \mathrm{fs}$ long, and a repetition rate of $1 \mathrm{kHz}$. Between each exposure, the beam was shifted laterally by $20 \mathrm{~nm}$.

beam with a circularly-polarized central one. Such structures can act as super-prisms near the 1D photonic stop-band.

In photo-structuring by dielectric breakdown, the material in the volume at the focus is fully ionized. Nonlinear absorption and avalanche multiplication of electrons in the focal region leads to the free-electron density reaching a critical density, at which point the material becomes reflective at the wavelength of irradiation. Then the dielectric breakdown ensues within a few optical cycles at intense irradiances. (For example, breakdown occurs at $7 \mathrm{TW} / \mathrm{cm}^{2} /$ pulse in silica. ${ }^{11}$ )

The dielectric breakdown is localized at the tip of the intensity profile and, in theory, it could be localized with atomic resolution because the classical diffraction limit is not applicable for the localization of nonlinear absorption. In reality, however, the resolution of $3 \mathrm{D}$ photo-structuring is determined by the depth of the absorption skin in the ionized focal volume. A unique feature of this method is that-within the enclosed focal volume-the absorbed energy can cause pressure far larger of the material's Young modulus. We expect to see new phases and new materials form in these high-pressure and high-temperature conditions. ${ }^{5}$

The 3D structures recorded in different materials have good prospects for use in biomedical research for 3D scaffolds and subsequent grafting, as well as for molecular and particle ratchets, micro-fluidic chips, and plasmonics. Photo-fabricated struc-

Continued on next page 

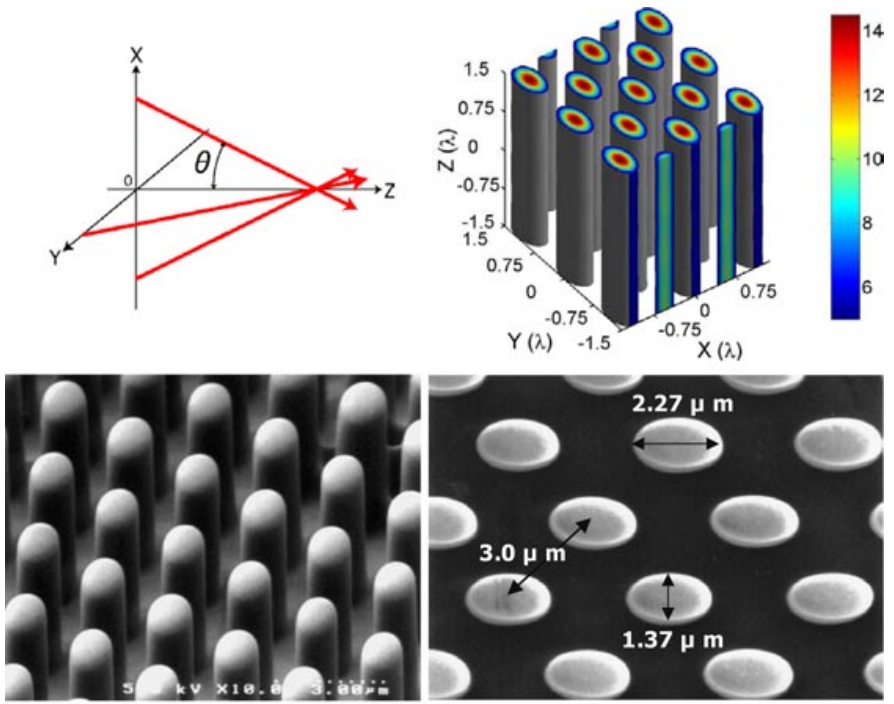

3D view

Top view

Figure 2. These beam alignment angles (at the top, left) were chosen to create a photonic crystal modelled at the top, right. The bottom images show the 2D photonic-crystal template fabricated holographically in SU-8 resist. The structure was made by $180 \mathrm{fs}$ pulses from an $800 \mathrm{~nm}$ waveleng th laser beam, at a pulse repetition rate of $1 \mathrm{kHz}$. The three beamlets converged at an angle $\theta=11^{\circ}$. The cumulative energy of the three beamlets was $19 \mu \mathrm{J}$ per pulse at the focus, and the exposure time was $120 \mathrm{~s}$.

tures are also likely to find use in photonics applications because they can be built to operate as waveguides, photonic crystals that operate in the IR, templates of photonic crystals that operate in the visible spectrum, gratings, or other diffractive elements.

\section{Author Information}

\section{Hiroaki Misawa and Saulius Juodkazis}

Hokkaido Univ.

Sapporo, Japan

Hiroaki Misawa is a Professor at the Research Institute for Electronic Science and Director of Nanotechnology Center at Hokkaido University. His research interests include photochemistry, light-matter interaction, ultra-fast processes in materials, photonic crystals, laser trapping, and plasmonics. He has authored more than 200 papers, including more than 25 presentations and invited talks at SPIE conferences.

Saulius Juodkazis is Associate Professor at the Research institute for Electronic Science of Hokkaido University. His current interests include space-time-spectrum-resolved characterization of light-matter interactions in the micro-domain, nano-photonics, plasmonics, laser tweezers, and applications of ultra-fast laser pulses. He has published over 90 scientific papers.
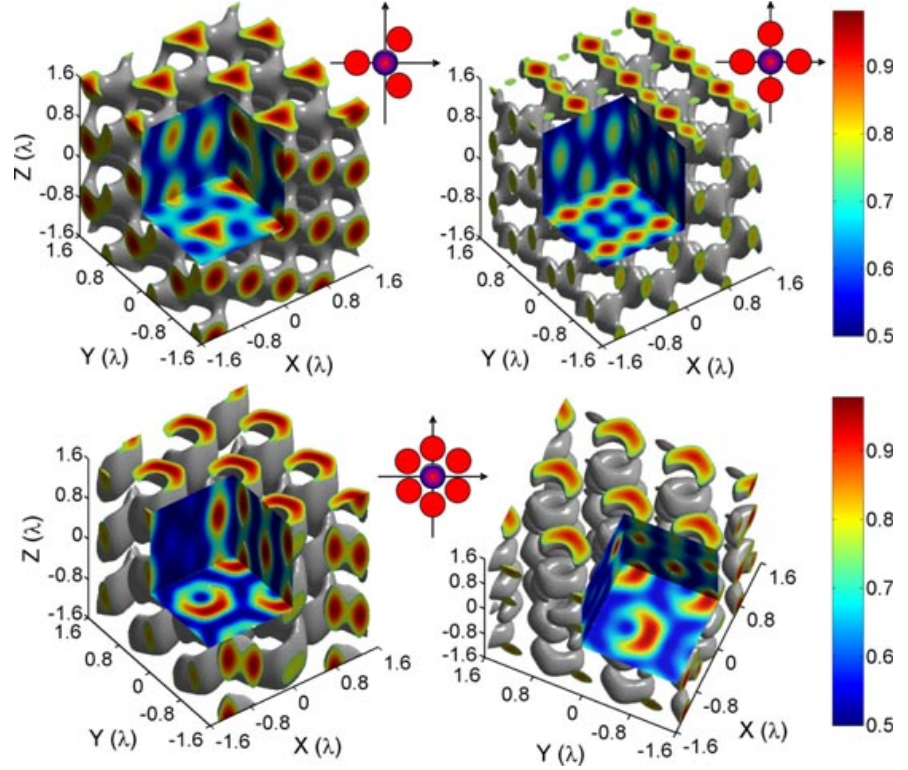

Figure 3. Three simulated intensity distributions of linearly-polarized three-,four-, and six-side-beams with acircularly-polarized (left-handed) central beam calculated as interference of plane waves. (Also, a pattern formed by six side-beams with the central and counter-propagatingcentral beam is given). The angle of the side beams with the central beam was $\theta=80^{\circ}$. The inset shows the beam alignment in the $x y$-plane.

\section{References}

1. M. Miwa, S. Juodkazis, T. Kawakami, S. Matsuo, and H. Misawa, Femtosecond two-photon stereo-lithography, Appl. Phys. A 73 (5), pp. 561-566, 2001

2. S. Juodkazis, V. Mizeikis, K. K. Seet, M. Miwa, and H. Misawa, Two-photon lithography of nanorods in SU-8 photoresist, Nanotechnol. 16, pp. 846-849, 2005.

3. K. K. Seet, V. Mizeikis, S. Matsuo, S. Juodkazis, and H. Misawa, Three-dimensional spiral - architecture photonic crystals obtained by direct laser writing, Adv. Mat. 17 (5), pp. 541-545, 2005. doi:10.1002/adma.200401527

4. K. K. Seet, V. Mizeikis, S. Juodkazis, and H. Misawa, Spiral three-dimensional photonic crystals for telecommunications spectral range, Appl. Phys. A, 2005 doi:10.1007/s00339-005-3459-y

5. S. Juodkazis, K. Nishimura, H. Misawa, T. Ebisui, R. Waki, S. Matsuo, and T. Okada, Control over the Crystalline State of Sapphire, Adv. Mat. 18 (11), pp. 1361 1364, 2006. doi:10.1002/adma.200501837

6. T. Kondo, S. Matsuo, S. Juodkazis, and H. Misawa, A novel femtosecond laser interference technique with diffractive beam splitter for fabrication of three-dimensional photonic crystals, Appl. Phys. Lett. 79 (6), pp. 725-727, 2001.

7. T. Kondo, S. Matsuo, S. Juodkazis, V. Mizeikis, and H. Misawa, Multiphoton fabrication of periodic structures by multibeam interference of femtosecond pulses, Appl. Phys. Lett. 82 (17), pp. 2758-2760, 2003.

8. S. Matsuo, S. Juodkazis, and H. Misawa, Femtosecond laser microfabrication of periodic structures using a microlens array, Appl. Phys. A 80 (4), pp. 683-685, 2004 doi:10.1007/s00339-004-3108-x

9. T. Kondo, S. Matsuo, S. Juodkazis, V. Mizeikis, and H. Misawa, Three-dimensional recording by femtosecond pulses in polymer materials, J. Photopolym. Sci. Tech. 16 (3), pp. $427-432,2003$

10. K. K. Seet, V. Jarutis, S. Juodkazis, and H. Misawa, Nanofabrication by direct laser writing and holography (invited paper), Proc. SPIE 6050, 2005. doi:10.1117/12.660605 11. O. Efimov, S. Juodkazis, and H. Misawa, Intrinsic single and multiple pulse laserinduced damage in silicate glasses in the femtosecond-to-nanosecond region, Phys. Rev. A 69, p. 042903, 2004.

(C) 2006 SPIE-The International Society for Optical Engineering 